\title{
Asymptotic Behavior of the Coupled Nonlinear Schrödinger Lattice System
}

\author{
Hengyan $\mathrm{Li}^{1}$ and Xin $\mathrm{Zhao}^{2}$ \\ ${ }^{1}$ School of Mathematics and Information Sciences, North China University of Water Resources and Electric Power, \\ Zhengzhou 450011, China \\ ${ }^{2}$ College of Information Technology, Jilin Agricultural University, Changchun 130118, China
}

Correspondence should be addressed to Hengyan Li; hengyanli989@126.com

Received 20 July 2014; Accepted 11 August 2014; Published 19 August 2014

Academic Editor: Yong Ren

Copyright (C) $2014 \mathrm{H}$. Li and X. Zhao. This is an open access article distributed under the Creative Commons Attribution License, which permits unrestricted use, distribution, and reproduction in any medium, provided the original work is properly cited.

This paper studies asymptotic behavior of solutions for the coupled nonlinear Schrödinger lattice system. We obtain the existence and stability of compact attractor by means of tail estimates method and finite-dimensional approximations.

\section{Introduction}

In the present paper, we consider the following dissipative coupled Schrödinger lattice system:

$$
\begin{aligned}
& i\left(\epsilon \dot{u}_{k}+\sigma_{1} u_{k}\right)+\epsilon(\mathscr{A} u)_{k}-V^{1} u_{k}+V^{2} v_{k} \\
& \quad+a_{1}\left|u_{k}\right|^{2} u_{k}+a_{2}\left|v_{k}\right|^{2} u_{k}=g_{k}^{1}, \\
& i\left(\epsilon \dot{v}_{k}+\sigma_{2} v_{k}\right)+\epsilon(\mathscr{A} v)_{k}-V^{3} v_{k}+V^{2} u_{k} \\
& \quad+a_{3}\left|v_{k}\right|^{2} v_{k}+a_{2}\left|u_{k}\right|^{2} v_{k}=g_{k}^{2},
\end{aligned}
$$

with initial condition

$$
u_{k}(0)=u_{k, 0}, \quad v_{k}(0)=v_{k, 0},
$$

where $k \in \mathbb{Z} ; \sigma_{1}, \sigma_{2}>0, V^{i}$ denotes a bounded positive potential with $i=1,2,3 ; g^{1}=\left(g_{k}^{1}\right)_{k \in \mathbb{Z}}, g^{2}=\left(g_{k}^{2}\right)_{k \in \mathbb{Z}} \in \ell^{2}$ denote external forces.

Nonlinear coupled Schödinger lattice system (1) can be seen as a discretization model of the two-component system of time-dependent nonlinear Gross-Pitaevskii equations. Gross-Pitaevskii equation arises quite naturally in a binary mixture of Bose-Einstein condensates with two different hyperfine states [1]. There are many analytical and numerical results on solitary wave solutions for this system (see [2-11]).

The study of the existence of compact attractor for general infinite lattice system can date back to Bates et al. [12], who used the tail estimates method to prove the asymptotic compactness of dissipative lattice system and the existence of compact attractor. For more general results on the existence of compact attractor for infinite lattice system, one can see [13]. Karachalios and Yannacopoulos [14] studied the asymptotic behavior of single nonlinear discrete Schrödinger equations.

We state our main results in this paper.

Theorem 1. The semigroup $\{\mathcal{S}(t)\}_{t \geq 0}$ generated by system (4) possesses a global attractor $\mathscr{A} \subset \ell^{2} \times \ell^{2}$ which is compact, connected, and maximal among the functional invariant sets in $\ell^{2} \times \ell^{2}$.

Theorem 2. The global attractor $\mathscr{A}_{N}$ converges $\mathscr{A}$ in the sense of the Hausdorff semidistance related to $\ell^{2} \times \ell^{2}$; that is,

$$
\lim _{N \rightarrow \infty} d\left(\mathscr{A}_{N}, \mathscr{A}\right)=0
$$

where $d\left(A_{1}, A_{2}\right)=\sup _{x \in A_{1}} \inf _{x \in A_{2}} d_{X}(x, y)$, for any nonempty compact subsets $A_{1}$ and $A_{2}$ in a metric space $\mathscr{X}$.

This paper is organized as follows. In the next section, we prove the global existence of the dissipative coupled Schrödinger lattice system (1). In Section 3, we show the stability of the global attractor. 


\section{Existence of the Global Attractor}

This section shows the existence of compact attractor of system (1) in $\ell^{2} \times \ell^{2}$. We denote a Hilbert space by $\ell^{2}$ with the scalar product $\langle u, v\rangle=\sum_{k \in \mathbb{Z}} u_{k} \bar{v}_{k}, u, v \in \ell^{2}$.

Firstly, we prove the global existence of solution of system (1). For convenience, we take the scalar form of system (1):

$$
\begin{gathered}
i\left(\epsilon \dot{u}+\sigma_{1} u\right)+\epsilon(\mathscr{A} u)-V^{1} u+V^{2} v \\
+a_{1}|u|^{2} u+a_{2}|v|^{2} u=g^{1}, \\
i\left(\epsilon \dot{v}+\sigma_{2} v\right)+\epsilon(\mathscr{A} v)-V^{3} v+V^{2} u \\
+a_{3}|v|^{2} v+a_{2}|u|^{2} v=g^{2},
\end{gathered}
$$

with initial condition $u(0)=u_{0}, v(0)=v_{0}$.

Let $H_{1}(u, v)=a_{1}|u|^{2}+a_{2}|v|^{2}$ and $H_{2}(u, v)=a_{3}|u|^{2}+a_{2}|v|^{2}$. Then system (4) can be rewritten as

$$
\begin{aligned}
& i\left(\epsilon \dot{u}+\sigma_{1} u\right)+\epsilon(\mathscr{A} u)-V^{1} u+V^{2} v+H_{1}(u, v) u=g^{1} \\
& i\left(\epsilon \dot{v}+\sigma_{2} v\right)+\epsilon(\mathscr{A} v)-V^{3} v+V^{2} u+H_{2}(u, v) v=g^{2} .
\end{aligned}
$$

Then we get the mild solution of (5) as

$$
\begin{aligned}
& u(t)=\mathscr{T}(t) u_{0}+i \epsilon^{-1} \int_{0}^{t} \mathscr{T}(t-s) F_{1}(u(s), v(s)) d s, \\
& v(t)=\mathscr{T}(t) v_{0}+i \epsilon^{-1} \int_{0}^{t} \mathscr{T}(t-s) F_{2}(u(s), v(s)) d s,
\end{aligned}
$$

where the semigroup $\mathscr{T}(t)=e^{i \mathscr{A} t}$ generated by the operator $i \mathscr{A}: \ell^{2} \rightarrow \ell^{2}$, and

$$
\begin{aligned}
& F_{1}(u, v):=i \sigma_{1} u-V^{1} u+V^{2} v+H_{1}(u, v) u-g^{1}, \\
& F_{2}(u, v):=i \sigma_{2} v-V^{3} v+V^{2} u+H_{2}(u, v) v-g^{2} .
\end{aligned}
$$

In order to prove the existence of compact attractor, we need the following proposition.

Proposition 3 (Hale [15], Temam [16]). Assume that $\mathscr{X}$ is a metric space and $\{\delta(t)\}_{t \geq 0}$ is a semigroup of continuous operators in $\mathscr{X}$. If $\{\mathcal{S}(t)\}_{t \geq 0}$ has an absorbing set and is asymptotically compact, then $\{\delta(t)\}_{t \geq 0}$ process a global attractor.

It is easy to verify that $F_{1}(u, v)$ and $F_{2}(u, v)$ satisfy the Lipschitz continuous property on any bounded set in $\ell^{2} \times \ell^{2}$. Using the same method in [14], we obtain the following result.

Theorem 4. Let $(u(0), v(0)) \in \ell^{2} \times \ell^{2}$. Then system (1) possesses a unique solution $(u(t), v(t)) \in \mathbb{C}^{1}\left([0, \widetilde{T}) ; \ell^{2} \times \ell^{2}\right)$ for some $\widetilde{T}>0$. If $\widetilde{T}<+\infty$, then

$$
\lim _{t \rightarrow \widetilde{T}}\|u, v\|_{\ell^{2} \times \ell^{2}}^{2}=+\infty .
$$

Lemma 5. Let $\left(u_{0}, v_{0}\right) \in \ell^{2} \times \ell^{2}$ and $g^{1}, g^{2} \in \ell^{2}$. Assume that $\sigma_{1}, \sigma_{2}>4$ holds. Then there exists a bounded absorbing ball $\mathscr{B}$ of the semigroup $\{\mathcal{S}(t)\}_{t \geq 0}$ generated by system (4) in $\ell^{2} \times \ell^{2}$. The radius of $\mathscr{B}$ is $\varrho>0$. Therefore, there exists $t_{0} \geq 0$ depending on $\mathscr{B}$ such that

$$
\mathcal{S}(t) \mathscr{B} \subset \mathscr{B}, \quad \forall t \geq t_{0} .
$$

Proof. Taking the imaginary part of the inner product of the first equation and second equation of (4) with $u(t)$ and $v(t)$, respectively, we have

$$
\begin{aligned}
& \frac{\epsilon}{2} \frac{d}{d t}\|u\|^{2}+\sigma_{1}\|u\|^{2}+V^{2} \operatorname{Im}\langle v, u\rangle=\operatorname{Im}\left\langle g^{1}, u\right\rangle, \\
& \frac{\epsilon}{2} \frac{d}{d t}\|v\|^{2}+\sigma_{2}\|v\|^{2}+V^{2} \operatorname{Im}\langle u, v\rangle=\operatorname{Im}\left\langle g^{2}, v\right\rangle .
\end{aligned}
$$

Summing up (10),

$$
\begin{gathered}
\frac{\epsilon}{2} \frac{d}{d t}\left(\|u\|^{2}+\|v\|^{2}\right)+\sigma_{1}\|u\|^{2}+\sigma_{2}\|v\|^{2} \\
=\operatorname{Im}\left\langle g^{1}, u\right\rangle+\operatorname{Im}\left\langle g^{2}, v\right\rangle .
\end{gathered}
$$

Let $C=\min \left\{\sigma_{1} / 2, \sigma_{2} / 2\right\}$. By Young inequality, we have

$$
\begin{aligned}
& \frac{d}{d t}\left(\|u\|^{2}+\|v\|^{2}\right)+\frac{2 C}{\epsilon}\left(\|u\|^{2}+\|v\|^{2}\right) \\
& \leq \frac{4}{\epsilon \sigma_{1}}\left\|g^{1}\right\|^{2}+\frac{4}{\epsilon \sigma_{2}}\left\|g^{2}\right\|^{2} .
\end{aligned}
$$

Applying Gronwall Lemma to (12), we have

$$
\begin{aligned}
\|u\|^{2}+\|v\|^{2} \leq & \left(\left\|u_{0}\right\|^{2}+\left\|v_{0}\right\|^{2}\right) e^{-(2 C / \epsilon) t} \\
& +\left(\frac{4}{\epsilon \sigma_{1}}\left\|g^{1}\right\|^{2}+\frac{4}{\epsilon \sigma_{2}}\left\|g^{2}\right\|^{2}\right) \\
& \times\left[\frac{2 C^{-1}}{\epsilon}-\frac{2 C^{-1}}{\epsilon} \exp \left(-\frac{2 C}{\epsilon} t\right)\right], \quad t \geq 0 .
\end{aligned}
$$

It implies that the semigroup $\{\delta(t)\}_{t \geq 0}$ possesses a bounded absorbing ball $\mathscr{B} \subset \ell^{2} \times \ell^{2}$ centered at 0 with radius $\varrho=$ $\sqrt{\left(2 / C \sigma_{1}\right)\left\|g^{1}\right\|^{2}+\left(2 / C \sigma_{2}\right)\left\|g^{2}\right\|^{2}}$.

Lemma 6. Assume that $\left(u_{0}, v_{0}\right) \in \mathscr{B}, g^{1}, g^{2} \in \ell^{2}$, and $\sigma_{1}$, $\sigma_{2}>4$. Then, there exist $T(\varepsilon)$ and $N(\varepsilon)$ such that the solution $(u(t), v(t))$ of system (4) satisfies

$$
\sum_{|k|>N(\varepsilon)}\left(\left|u_{k}\right|^{2}+\left|v_{k}\right|^{2}\right) \leq \varepsilon, \quad \forall t \geq T(\varepsilon) .
$$

Proof. Define $\eta(x) \in \mathbb{C}\left(\mathbb{R}^{+} ;[0,1]\right)$ by

$$
\begin{aligned}
& \eta(x)=0, \quad \forall x \in[0,1], \\
& \eta(x)=1, \quad \forall x \in[2,+\infty), \\
& \left|\eta^{\prime}(x)\right| \leq \eta_{0},
\end{aligned}
$$

where $\eta_{0}$ is a positive constant number. 
Taking the imaginary part of the inner product of the first equation and second equation of (4) with $\overline{\widetilde{u}}_{k}:=\eta(|k| / M) \bar{u}_{k}$ and $\overline{\widetilde{v}}_{k}:=\eta(|k| / M) \bar{v}_{k}$ in $\ell^{2}$, respectively, where $M \in \mathbb{Z}$ is large enough, we have

$$
\begin{aligned}
& \frac{\epsilon}{2} \frac{d}{d t} \sum_{k \in \mathbb{Z}} \eta\left(\frac{|k|}{M}\right)\left|u_{k}\right|^{2}+\sigma_{1} \sum_{k \in \mathbb{Z}} \eta\left(\frac{|k|}{M}\right)\left|u_{k}\right|^{2} \\
& +V^{2} \operatorname{Im} \sum_{k \in \mathbb{Z}} \eta\left(\frac{|k|}{M}\right) v_{k} \bar{u}_{k} \\
& \quad-\operatorname{Im} \sum_{k \in \mathbb{Z}}\left[(\mathscr{B} \overline{\tilde{u}})_{k}(\mathscr{B} u)_{k}\right]=\operatorname{Im} \sum_{k \in \mathbb{Z}} g_{k}^{1} \eta\left(\frac{|k|}{M}\right) \bar{u}_{k}, \\
& \frac{\epsilon}{2} \frac{d}{d t} \sum_{k \in \mathbb{Z}} \eta\left(\frac{|k|}{M}\right)\left|v_{k}\right|^{2}+\sigma_{1} \sum_{k \in \mathbb{Z}} \eta\left(\frac{|k|}{M}\right)\left|v_{k}\right|^{2} \\
& \quad+V^{2} \operatorname{Im} \sum_{k \in \mathbb{Z}} \eta\left(\frac{|k|}{M}\right) u_{k} \bar{v}_{k} \\
& \quad-\operatorname{Im} \sum_{k \in \mathbb{Z}}\left[(\mathscr{B} \overline{\tilde{v}})_{k}(\mathscr{B} v)_{k}\right]=\operatorname{Im} \sum_{k \in \mathbb{Z}} g_{k}^{2} \eta\left(\frac{|k|}{M}\right) \bar{v}_{k} .
\end{aligned}
$$

Summing up (16), we get

$$
\begin{aligned}
& \frac{\epsilon}{2} \frac{d}{d t} \sum_{k \in \mathbb{Z}} \eta\left(\frac{|k|}{M}\right)\left(\left|u_{k}\right|^{2}+\left|v_{k}\right|^{2}\right) \\
& \quad+\sigma_{1} \sum_{k \in \mathbb{Z}} \eta\left(\frac{|k|}{M}\right)\left|u_{k}\right|^{2}+\sigma_{2} \sum_{k \in \mathbb{Z}} \eta\left(\frac{|k|}{M}\right)\left|v_{k}\right|^{2} \\
& =\operatorname{Im} \sum_{k \in \mathbb{Z}}\left[(\mathscr{B} \overline{\widetilde{u}})_{k}(\mathscr{B} u)_{k}+(\mathscr{B} \overline{\tilde{v}})_{k}(\mathscr{B} v)_{k}\right] \\
& \quad+\operatorname{Im} \sum_{k \in \mathbb{Z}}\left[g_{k}^{1} \eta\left(\frac{|k|}{M}\right) \bar{u}_{k}+g_{k}^{2} \eta\left(\frac{|k|}{M}\right) \bar{v}_{k}\right] .
\end{aligned}
$$

Let $\sigma=\min \left\{\sigma_{1} / 2, \sigma_{2} / 2\right\}>2$. By Cauchy-Schwartz inequality and (17), we have

$$
\begin{aligned}
& \frac{d}{d t} \sum_{k \in \mathbb{Z}} \eta\left(\frac{|k|}{M}\right)\left(\left|u_{k}\right|^{2}+\left|v_{k}\right|^{2}\right) \\
& \quad+\frac{2(\sigma-2)}{\epsilon} \sum_{k \in \mathbb{Z}} \eta\left(\frac{|k|}{M}\right)\left(\left|u_{k}\right|^{2}+\left|v_{k}\right|^{2}\right) \\
& \leq \frac{2}{\epsilon \sigma} \sum_{|k| \geq M}\left(\left|g_{k}^{1}\right|^{2}+\left|g_{k}^{2}\right|^{2}\right) .
\end{aligned}
$$

Applying Gronwall Lemma, we can obtain

$$
\begin{aligned}
\sum_{|k|>M} & \left(\left|u_{k}\right|^{2}+\left|v_{k}\right|^{2}\right) \\
\leq & e^{-(2(\sigma-2) / \epsilon)\left(t-t_{0}\right)} \sum_{|k|>M}\left(\left|u_{k}\left(t_{0}\right)\right|^{2}+\left|v_{k}\left(t_{0}\right)\right|^{2}\right) \\
+ & \frac{2}{\epsilon \sigma} \sum_{|k|>M}\left(\left|g_{k}^{1}\right|^{2}+\left|g_{k}^{2}\right|^{2}\right) \\
\times & {\left[\left(\frac{2(\sigma-2)}{\epsilon}\right)^{-1}-\left(\frac{2(\sigma-2)}{\epsilon}\right)^{-1}\right.} \\
& \left.\times e^{-(2(\sigma-2) / \epsilon)\left(t-t_{0}\right)}\right]
\end{aligned}
$$

where $t_{0}$ and $\varrho$ are the time of entry of initial data bounded in $\ell^{2} \times \ell^{2}$ and radius of the absorbing ball in $\ell^{2} \times \ell^{2}$.

Since $g^{1}, g^{2} \in \ell^{2}$, then, for any given $\epsilon>0$ and $t>t_{0}$, there exist $N(\epsilon)$ and $T(\epsilon)$ such that

$$
\sum_{|k|>N}\left(\left|u_{k}\right|^{2}+\left|v_{k}\right|^{2}\right) \leq C(\sigma) \varepsilon, \quad t>T(\epsilon),
$$

where $C(\sigma)$ denote a constant number depending on $\sigma$.

Lemma 7. The semigroup $\{\mathcal{S}(t)\}_{t>0}$ is asymptotically compact in $\ell^{2} \times \ell^{2}$; that is, if sequences $\left\{u_{k}\right\}_{k \in \mathbb{Z}},\left\{v_{k}\right\}_{k \in \mathbb{Z}}$ are bounded in $\ell^{2}$ and $t_{k} \rightarrow \infty$, then $\mathcal{S}\left(t_{k}\right)\left(u_{k}, v_{k}\right)$ is precompact in $\ell^{2} \times \ell^{2}$.

Proof. Define $\Gamma=\left\{\delta\left(t_{k}\right)\left(u_{k}, v_{k}\right):\left(u_{k}, v_{k}\right) \in \mathscr{B}, t_{k} \rightarrow\right.$ $\infty$ as $k \rightarrow \infty$. Our purpose is to prove that $\Gamma$ has finite covering balls of radii $\varepsilon$.

By Lemma 6, we know that, for all $\left(u_{k}, v_{k}\right) \in \Gamma$, there exist $N(\varepsilon)$ and $T(\varepsilon)$ such that

$$
\sum_{|k|>N(\varepsilon)}\left(\left|u_{k}\right|^{2}+\left|v_{k}\right|^{2}\right) \leq \frac{\varepsilon}{2}, \quad t \geq T(\varepsilon) .
$$

We consider the set $\widetilde{\Gamma}=\left\{\left(u_{k}, v_{k}\right)_{|k| \leq N(\varepsilon)}:\left(u_{k}, v_{k}\right) \in \Gamma\right\}$ in $\mathbb{R}^{2 N(\varepsilon)+1}$. Note that $\widetilde{\Gamma}$ is bounded in $\mathbb{R}^{2 N(\varepsilon)+1}$, so it is precompact in $\mathbb{R}^{2 N(\varepsilon)+1}$; that is, there exists a family of balls of radii $\varepsilon / 2$, which covers $\widetilde{\Gamma}$. This together with (21) implies that the set $\Gamma$ has finite covering balls of radii $\varepsilon$. This completes the proof.

Therefore, by Lemmas 6 and 7 and Proposition 3, we conclude that Theorem 1 holds.

\section{Finite Approximation of the Global Attractor}

In this section, we study the stability of global attractor of lattice dynamical system generated by (1)-(2) under its approximation by a global attractor of an appropriate infinite dimensional dynamical system. 
We consider the following finite dimensional boundary value problem:

$$
\begin{gathered}
i\left(\epsilon \dot{u}_{k}+\sigma_{1} u_{k}\right)+\epsilon\left(\mathscr{A} u_{k}\right)-V^{1} u_{k}+V^{2} v_{k} \\
-a_{1}\left|u_{k}\right|^{2} u_{k}-a_{2}\left|v_{k}\right|^{2} u_{k}=g_{k}^{1}, \\
i\left(\epsilon \dot{v}_{k}+\sigma_{2} v_{k}\right)+\epsilon\left(\mathscr{A} v_{k}\right)-V^{3} v_{k}+V^{2} u_{k} \\
-a_{3}\left|v_{k}\right|^{2} v_{k}-a_{2}\left|u_{k}\right|^{2} v_{k}=g_{k}^{2}, \\
u_{-N-1}(t)=u_{N+1}(t)=0, \quad v_{-N-1}(t)=v_{N+1}(t)=0, \\
u_{k}(0)=u_{k, 0}, \quad v_{k}(0)=v_{k, 0} .
\end{gathered}
$$

In similar process with infinite dimensional problem (1)(2), we have the following well-posedness and asymptotic behavior of finite dimensional system (22).

Lemma 8. Let $u_{0}:=\left(u_{k, 0}\right)_{k \leq N}, v_{0}:=\left(v_{k, 0}\right)_{k \leq N} \in \mathbb{C}^{2 N+1}$. Assume that $\sigma_{1}, \sigma_{2}>4$ holds. Then there exists a unique solution $(\widetilde{u}, \widetilde{v}) \in \mathbb{C}^{1}\left([0, \infty), \mathbb{C}^{2 N+1} \times \mathbb{C}^{2 N+1}\right)$. The dynamical system $\mathcal{S}_{N}(t)$ generated by (22) possesses a bounded absorbing set $\mathscr{B}_{N} \in \mathbb{C}^{2 N+1} \times \mathbb{C}^{2 N+1}$ and global attractor $\mathscr{A}_{N} \subset \mathscr{B}_{N}$.

In order to verify the global attractor $\mathscr{A}$ of semigroup $\delta(t)$ being approximated by the global attractor $\mathscr{A}_{N}$ of semigroup $\mathcal{S}_{N}(t)$ as $N \rightarrow \infty$, we extend the solution of (22) to infinite dimensional space $\ell^{2} \times \ell^{2}$, as

$$
\begin{aligned}
& \left(u_{N}(t), v_{N}(t)\right)_{N \in \mathbb{Z}} \\
& \quad:= \begin{cases}(\widetilde{u}(t), \widetilde{v}(t)):=\left(u_{k}(t), v_{k}(t)\right)_{|k| \leq N}, & |k| \leq N, \\
0, & |k|>N .\end{cases}
\end{aligned}
$$

Proof of Theorem 2. We denote global attractors generated by semigroups $\mathcal{S}(t)$ and $\mathcal{S}_{N}(t)$ by $\mathscr{A}$ and $\mathscr{A}_{N}$, respectively. By Lemma 7, we can get that $\widetilde{\mathscr{B}}:=\mathscr{B} \cap\left(\mathbb{C}^{2 N+1} \times \mathbb{C}^{2 N+1}\right)$ is also an absorbing set for $\mathcal{S}_{N}(t)$. Then, we have

$$
\begin{array}{r}
\mathscr{A}_{N} \subset \widetilde{\mathscr{B}} \subset \mathscr{B}_{0}:=\mathcal{O} \bigcap\left(\mathbb{C}^{2 N+1} \times \mathbb{C}^{2 N+1}\right), \\
\mathscr{A}_{N} \text { attracts } \mathscr{B}_{0},
\end{array}
$$

where $\mathcal{O}$ denotes an open neighborhood of absorbing ball $\mathscr{B}$. In light of Theorem 6.1 in [14], for obtaining (3), we only need to verify that, for every compact interval $J$ of $\mathbb{R}^{+}$,

$$
\sup _{\left(u_{0}, v_{0}\right) \in \mathscr{B}_{0}} \sup _{t \in J} d\left(\mathcal{S}_{N}(t)\left(u_{0}, v_{0}\right), \mathcal{S}(t)\left(u_{0}, v_{0}\right)\right) \longrightarrow 0,
$$

$$
\text { as } N \longrightarrow \infty \text {. }
$$

Let $(\widetilde{u}(t), \widetilde{v}(t))=\mathcal{S}_{N}(t)\left(u_{0}, v_{0}\right) \in \mathbb{C}^{2 N+1} \times \mathbb{C}^{2 N+1}$ be a solution of problem (22). Since $(\widetilde{u}(t), \widetilde{v}(t)) \in \mathscr{B}_{N}$, for every $t \in \mathbb{R}^{+}$. If we denote $\varrho_{N}$ as the radius of absorbing ball $\mathscr{B}_{N}$, by (23) and (22), we have

$$
\begin{gathered}
\left\|\left(u_{N}(t), v_{N}(t)\right)\right\|_{\ell^{2} \times \ell^{2}} \leq \varrho_{N}^{2}, \\
\left\|\left(\dot{u}_{N}(t), \dot{v}_{N}(t)\right)\right\|_{\ell^{2} \times \ell^{2}} \leq C_{5}\left(\varrho_{N},\left\|g^{1}\right\|^{2},\left\|g^{1}\right\|^{2}\right),
\end{gathered}
$$

which implies that, for every $t \in J$, there exists a subsequence of $\left(u_{N}(t), v_{N}(t)\right)$ (still denoted by itself) such that

$$
\begin{array}{r}
\left(u_{N}(t), v_{N}(t)\right) \longrightarrow(u(t), v(t)), \\
\text { weakly in } \mathbb{C}\left(J, \ell^{2} \times \ell^{2}\right), \\
\left(u_{N}(t), v_{N}(t)\right) \longrightarrow(u(t), v(t)), \\
\text { weakly star in } \mathbb{L}^{\infty}\left(J, \ell^{2} \times \ell^{2}\right), \\
\left(\dot{u}_{N}(t), \dot{v}_{N}(t)\right) \longrightarrow(\dot{u}(t), \dot{v}(t)), \\
\text { weakly star in } \mathbb{L}^{\infty}\left(J, \ell^{2} \times \ell^{2}\right) .
\end{array}
$$

Next, according to Theorem 6.1 in [14], for proving (25), it suffices to show that solution $\left(u_{N}(t), v_{N}(t)\right)$ of system $(22)$ converges to solution $(u(t), v(t))$ of system (1) in any compact interval $J$ of $\mathbb{R}^{+}$and $\left(u_{0}, v_{0}\right)$ in a bounded set of $\ell^{2} \times \ell^{2}$.

By (26), we can define differentiable functions

$$
\begin{array}{r}
\Upsilon_{N}^{1}(t):=\left(u_{N}(t), \phi\right), \quad \Upsilon_{N}^{2}(t):=\left(v_{N}(t), \phi\right), \\
\forall \phi
\end{array}
$$

Then,

$$
\left(\Upsilon_{N}^{1}(t)\right)^{\prime}:=\left(\dot{u}_{N}(t), \phi\right), \quad\left(\Upsilon_{N}^{2}(t)\right)^{\prime}:=\left(\dot{v}_{N}(t), \phi\right) .
$$

It follows from the mean-value theorem that there exist $\zeta_{1}, \zeta_{2} \in J$ such that, for any fixed $t, s \in J$,

$$
\begin{aligned}
& \left|\left(u_{N}(t)-u_{N}(s), \phi\right)\right| \\
& \quad=\left|\Upsilon_{N}^{1}(t)-\Upsilon_{N}^{1}(s)\right| \\
& \quad=\left|\left(\dot{u}_{N}(t), \phi\right)\right||t-s| \leq C_{5}|t-s|, \\
& \quad\left|\left(v_{N}(t)-v_{N}(s), \phi\right)\right| \\
& \quad=\left|\Upsilon_{N}^{2}(t)-\Upsilon_{N}^{2}(s)\right| \\
& \quad=\left|\left(\dot{v}_{N}(t), \phi\right)\right||t-s| \leq C_{6}|t-s| .
\end{aligned}
$$

Hence, there exists a constant $C_{7}$ (independent of $N$ ) such that

$$
\left\|\left(u_{N}(t)-u_{N}(s), v_{N}(t)-v_{N}(s)\right)\right\|_{\ell^{2} \times \ell^{2}} \leq C_{7}|t-s|,
$$

which implies that the sequences $\left(\Upsilon_{N}^{1}, \Upsilon_{N}^{2}\right)$ are equicontinuous. This combines with (26) and Ascoli-Arzelá theorem; we get that the weak convergence in (27) is actually strong convergence.

We define

$$
\begin{aligned}
& \widetilde{F}_{1}(u, v):=-V^{1} u+V^{2} v-H_{1}(u, v) u, \\
& \widetilde{F}_{2}(u, v):=-V^{3} v+V^{2} u-H_{2}(u, v) v,
\end{aligned}
$$

where $H_{1}(u, v)$ and $H_{2}(u, v)$ are defined in (5). Similar to Lemma 5, we can get that $\widetilde{F}_{1}, \widetilde{F}_{2}: \ell^{2} \times \ell^{2} \rightarrow \ell^{2} \times \ell^{2}$ are 
Lipschitz continuous on any bounded sets in $\ell^{2} \times \ell^{2}$; that is, there exists constant $C_{8}\left(\varrho_{N}\right)$ such that

$$
\begin{aligned}
& \left\|\widetilde{F}_{1}\left(u_{N}, v_{N}\right)-\widetilde{F}_{1}(u, v)\right\| \leq C_{9}\left\|\left(u_{N}-u, v_{N}-u\right)\right\|_{\ell^{2} \times \ell^{2}}, \\
& \left\|\widetilde{F}_{2}\left(u_{N}, v_{N}\right)-\widetilde{F}_{2}(u, v)\right\|_{\ell^{2} \times \ell^{2}} \leq C_{8}\left\|\left(u_{N}-u, v_{N}-v\right)\right\|_{\ell^{2} \times \ell^{2}} .
\end{aligned}
$$

Then, by (27), $\forall \omega(t) \in \mathbb{C}_{0}^{\infty}(J)$, we have

$$
\begin{array}{r}
\left|\int_{J}\left(\widetilde{F}_{1}\left(u_{N}, v_{N}\right)-\widetilde{F}_{1}(u, v), \omega(t)\right) d t\right| \\
\text { as } N \longrightarrow 0 \\
\left|\int_{J}\left(\widetilde{F}_{2}\left(u_{N}, v_{N}\right)-\widetilde{F}_{2}(u, v), \omega(t)\right) d t\right| \\
\text { as } N \longrightarrow 0
\end{array}
$$

By the formula in [17, page 59], for $\phi \in \ell^{2}$, we get

$$
\begin{aligned}
& \int_{J}\left(i \dot{u}_{N}(t), \phi\right) \omega(t) d t+\epsilon \int_{J}\left(\mathscr{A} u_{N}, \phi\right) \omega(t) d t \\
& \quad+\int_{J}\left(\widetilde{F}_{1}\left(u_{N}(t), v_{N}(t)\right), \phi\right) \omega(t) d t \\
& =\int_{J}\left(g^{1}, \phi\right) \omega(t) d t \\
& \int_{J}\left(i \dot{v}_{N}(t), \phi\right) \omega(t) d t+\epsilon \int_{J}\left(\mathscr{A} v_{N}, \phi\right) \omega(t) d t \\
& \quad+\int_{J}\left(\widetilde{F}_{1}\left(u_{N}(t), v_{N}(t)\right), \phi\right) \omega(t) d t \\
& =\int_{J}\left(g^{2}, \phi\right) \omega(t) d t .
\end{aligned}
$$

Since $J$ is arbitrary, (36) holds for all $t \in \mathbb{R}^{+}$. By (27) and (36), we obtain that $(u(t), v(t))$ is a bounded solution of (1) and $(u(t), v(t)) \in \mathscr{A}$, which implies that $\left(u_{N}(0), v_{N}(0)\right) \rightarrow$ $(u(0), v(0))$. If the convergence holds for any other subsequence satisfying (36), then it contradicts uniqueness of the solution. Therefore, we deduce that the convergence holds for original sequence $\left(u_{N}, v_{N}\right)$, which implies that (25) holds. This completes the proof.

Remark 9. We have discussed the existence of compact attractor for the one dimensional coupled Schrödinger lattice. By the same method, we also can obtain the same result for spatial discretization of coupled Schrödinger system in higher dimensional $\mathbb{Z}^{n}, n \geq 2$. It takes the following form:

$$
\begin{gathered}
i\left(\epsilon \dot{u}_{k}+\sigma_{1} u_{k}\right)+\epsilon(\mathscr{A} u)_{k}-V^{1} u_{k}+V^{2} v_{k} \\
-a_{1}\left|u_{k}\right|^{2} u_{k}-a_{2}\left|v_{k}\right|^{2} u_{k}=g_{k}^{1}, \\
i\left(\epsilon \dot{v}_{k}+\sigma_{2} v_{k}\right)+\epsilon(\mathscr{A} v)_{k}-V^{3} v_{k}+V^{2} u_{k} \\
-a_{3}\left|v_{k}\right|^{2} v_{k}-a_{2}\left|u_{k}\right|^{2} v_{k}=g_{k}^{2},
\end{gathered}
$$

where $\epsilon>0$ is a small parameter, $k=\left(k_{1}, k_{2}, \ldots, k_{n}\right) \in \mathbb{Z}^{n}$, $a_{i}>0, V^{i}$ are constant potential $(i=1,2,3)$, and $\mathscr{A}$ is the discrete Laplacian operator defined as

$$
\begin{aligned}
(\mathscr{A} u)_{k \in \mathscr{Z}^{n}}= & u_{\left(k_{1}-1, k_{2}, \ldots, k_{n}\right)}+u_{\left(k_{1}, k_{2}-1, \ldots, k_{n}\right)} \\
& +\cdots+u_{\left(k_{1}, k_{2}, \ldots, k_{n}-1\right)} \\
& +u_{\left(k_{1}+1, k_{2}, \ldots, k_{n}\right)}+u_{\left(k_{1}, k_{2}+1, \ldots, k_{n}\right)} \\
& +\cdots+u_{\left(k_{1}, k_{2}, \ldots, k_{n}+1\right)}-2 n u_{\left(k_{1}, k_{2}, \ldots, k_{n}\right)}
\end{aligned}
$$

Define operators $\mathbb{B}_{\gamma}, \mathbb{B}_{\gamma}^{*}: \ell^{2} \rightarrow \ell^{2}$ as

$$
\begin{aligned}
& \left(\mathbb{B}_{\nu} u\right)_{k \in \mathbb{Z}^{n}}=u_{\left(k_{1}, \ldots, k_{\nu-1}, k_{v}+1, k_{v+1}, \ldots, k_{n}\right)}-u_{\left(k_{1}, \ldots, k_{n}\right)}, \\
& \left(\mathbb{B}_{\nu}^{*} u\right)_{k \in \mathbb{Z}^{n}}=u_{\left(k_{1}, \ldots, k_{\nu-1}, k_{v}-1, k_{v+1}, \ldots, k_{n}\right)}-u_{\left(k_{1}, \ldots, k_{n}\right)} .
\end{aligned}
$$

Then the operator $\mathscr{A}$ satisfies

$$
\begin{aligned}
(-\mathscr{A} u, v) & =\sum_{\nu=1}^{n}\left(\mathbb{B}_{\nu}^{*} \mathbb{B}_{\nu} u, v\right) \\
& =\sum_{\nu=1}^{n}\left(\mathbb{B}_{\nu} u, \mathbb{B}_{\nu} v\right), \quad \forall u, v \in \ell^{2} .
\end{aligned}
$$

\section{Conflict of Interests}

The authors declare that there is no conflict of interests regarding the publication of this paper.

\section{References}

[1] L. Pitaevskii and S. Stingari, Bose-Einstein Condensation, Oxford Univeristy Press, Oxford, UK, 2003.

[2] A. Ambrosetti and E. Colorado, "Standing waves of some coupled nonlinear Schrödinger equations," Journal of the London Mathematical Society, vol. 75, no. 1, pp. 67-82, 2007.

[3] K. W. Chow, "Periodic solutions for a system of four coupled nonlinear Schrödinger equations," Physics Letters A, vol. 285, no. 5-6, pp. 319-326, 2001.

[4] F. T. Hioe, "Solitary waves and $N$ coupled nonlinear equations," Journal of Physics A, vol. 32, no. 7, pp. 1217-1223, 1999.

[5] F. T. Hioe and T. S. Salter, "Special set and solutions of coupled nonlinear Schrödinger equations," Journal of Physics A: Mathematical and General, vol. 35, no. 42, pp. 8913-8928, 2002.

[6] G. M. N’Guérékata and A. Pankov, "Global well-posedness for discrete non-linear Schrödinger equation," Applicable Analysis, vol. 89, no. 9, pp. 1513-1521, 2010.

[7] P. Panayotaros and D. Pelinovsky, "Periodic oscillations of discrete NLS solitons in the presence of diffraction management," Nonlinearity, vol. 21, no. 6, pp. 1265-1279, 2008.

[8] A. Pankov, "Gap solitons in periodic discrete no nlinear schrodinger equations," Nonlinearity, vol. 19, no. 1, pp. 27-40, 2006.

[9] A. Pankov and V. Rothos, "Periodic and decaying solutions in discrete nonlinear Schrödinger with saturable nonlinearity," Proceedings of The Royal Society A, vol. 464, no. 2100, pp. 32193236, 2008. 
[10] V. M. Rothos and T. C. Bountis, "The dynamics of coupled perturbed discretized NLS equations," Physica D: Nonlinear Phenomena, vol. 113, no. 2-4, pp. 326-330, 1998.

[11] G. Zhang, "Breather solutions of the discrete nonlinear Schrödinger equations with unbounded potentials," Journal of Mathematical Physics, vol. 50, no. 1, Article ID 013505, 12 pages, 2009.

[12] P. W. Bates, K. Lu, and B. Wang, "Attractors for lattice dynamical systems," International Journal of Bifurcation and Chaos in Applied Sciences and Engineering, vol. 11, no. 1, pp. 143-153, 2001.

[13] B. X. Wang, "Dynamics of systems on infinite lattices," Journal of Differential Equations, vol. 221, no. 1, pp. 224-245, 2006.

[14] N. I. Karachalios and A. N. Yannacopoulos, "Global existence and compact attractors for the discrete nonlinear Schrödinger equation," Journal of Differential Equations, vol. 217, no. 1, pp. 88-123, 2005.

[15] J. K. Hale, Asymptotic Behavior of Dissipative Systems, vol. 25 of Mathematical Surveys and Monographs, American Mathematical Society, Providence, RI, USA, 1998.

[16] R. Temam, Infinite-Dimensional Dynamical Systems in Mechanics and Physics, vol. 68 of Applied Mathematical Sciences, Springer, New York, NY, USA, 2nd edition, 1997.

[17] T. Cazenave, An Introduction to Nonlinear Schrödinger Equations, vol. 26 of Textos des Metodos Matematicos, I.M.U.F.R.J., Rio de Janeiro, Brazil, 1996. 


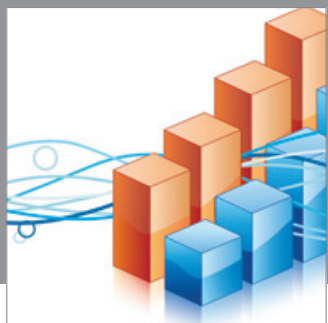

Advances in

Operations Research

mansans

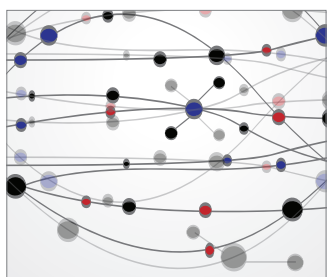

The Scientific World Journal
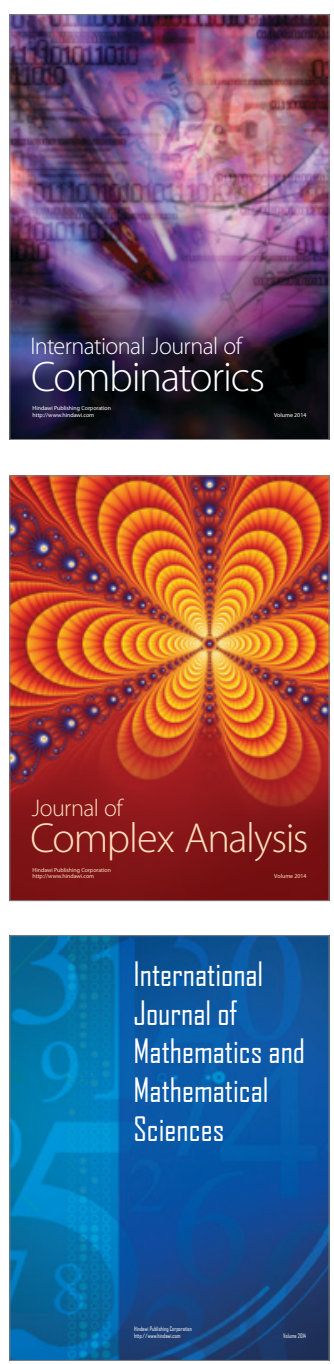
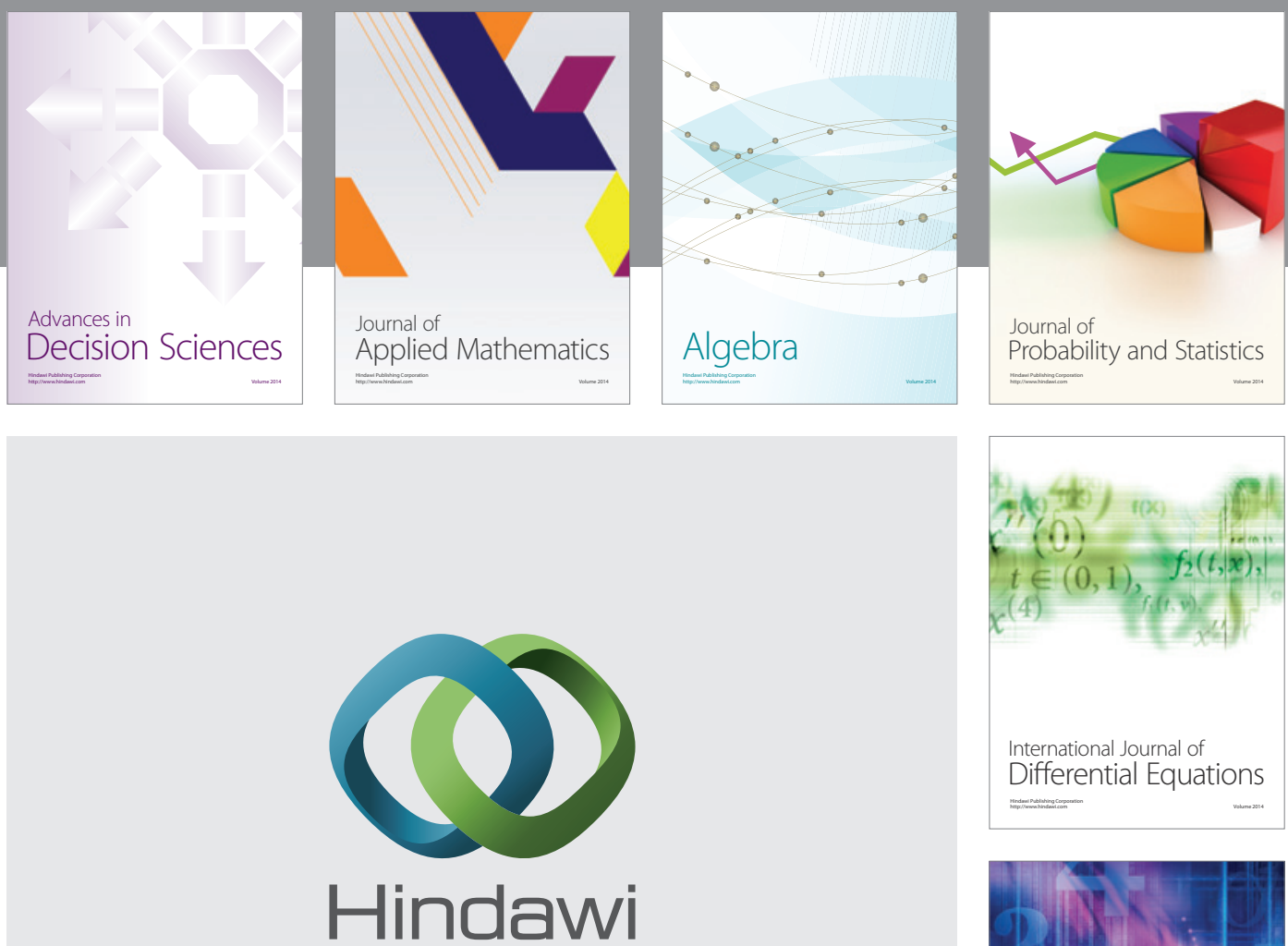

Submit your manuscripts at http://www.hindawi.com
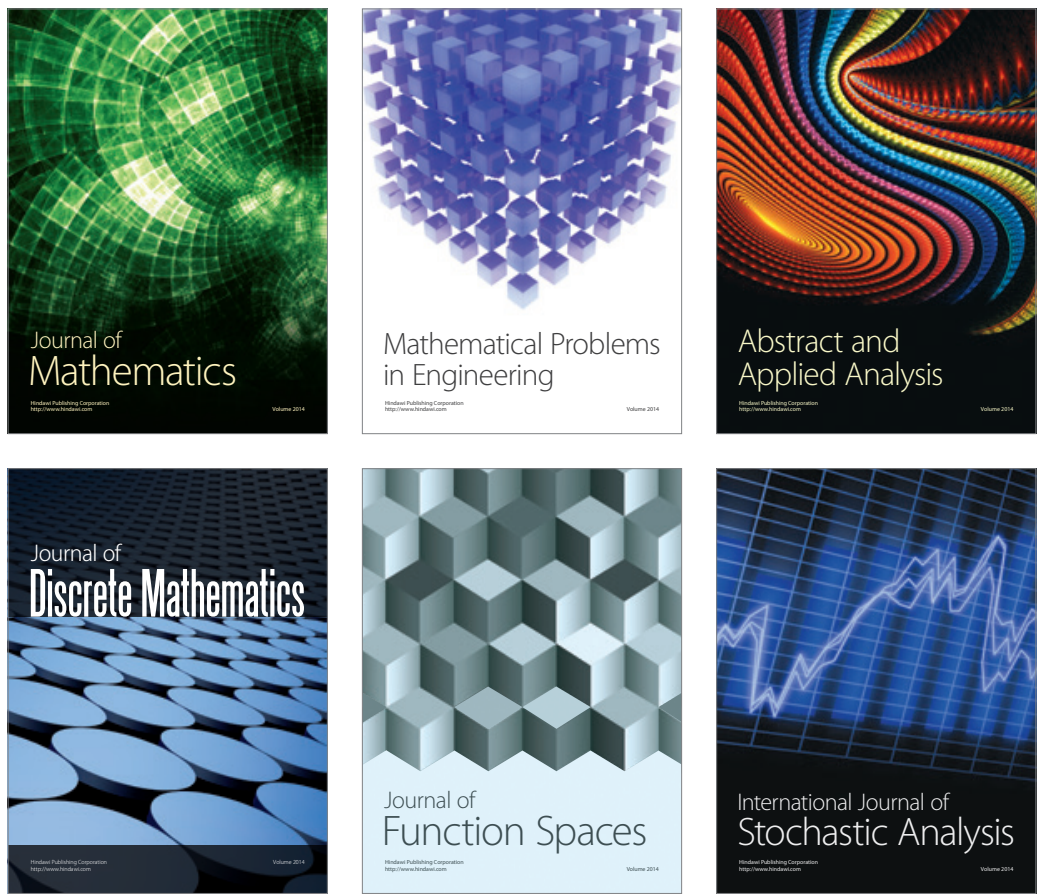

Journal of

Function Spaces

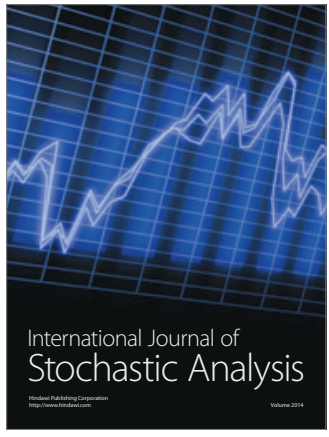

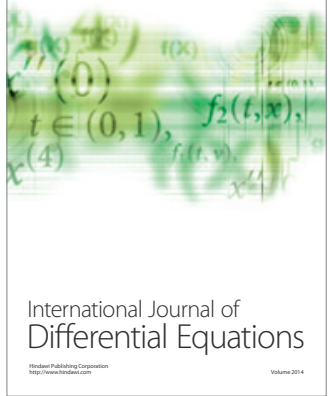
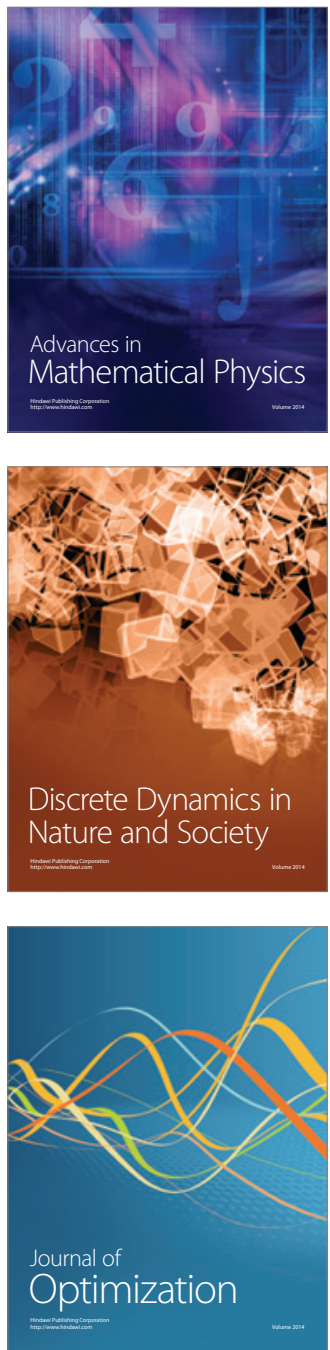